\title{
Evaluation of Prognostic Indicators in Non-Hodgkin Lymphomas: The Role of Apoptotic and Proliferative Indices
}

\author{
Chandhru Mari R, Shantaraman K* and JohnsyMerla J
}

Department of Pathology, Tirunelveli medical college, Tirunelveli, Tamilnadu, India

\section{ABSTRACT}

Background: Non Hodgkin lymphoma (NHL) is a collection of different subtypes each with different clinical presentations, treatment regimen and prognosis. To date there is no single classification that can predict how a particular tumour in an individual patient might behave. So a need to look at other prognostic indicators has become increasingly relevant. In this study various subtypes of NHL were studied. They were classified by working formulation classification and apoptotic \& proliferative indices which were taken as prognostic indicators were applied.

Methods: Histopathological tissue materials of NHL cases from the department of pathology ,tirunelveli medical college were collected and studied. All cases were classified by working formulation into low,intermediate, high grade. Apoptotic indices were calculated by counting apoptotic bodies per 1000 cells and expressed in percentage. Immunohistochemical stain for Ki67 was done . Proliferative indices were calculated by Ki67 positive cells per 1000 cells and expressed in percentage.

Results: Among the total 57 cases of NHL, by working formulation classification 21 cases are of low grade,23 cases are intermediate grade and 13 cases are high grade. And then by applying prognostic indicators out of the 21 cases of low grade lymphomas 4 cases show higher proliferative capacity. Similarly among the 23 cases of intermediate grade lymphomas 5 cases show lower proliferative capacity and other 5 cases with higher proliferative capacity.Among the 13 cases of high grade lymphoma all the cases shows a very higher proliferative capacity with very high apoptotic indices.

Conclusion: Inspite the availability of a number of new classification for NHL working formulation still holds good in most cases. Though apoptotic indices were found not to be significant in this study,proliferative indices were found to play an important in identifying the aggressiveness of tumour behaviour. The outcome of the analysis of these indices has a positive correlation and has a definite impact over the prognosis of patients with NHL.

Keywords: Non Hodgkin Lymphoma, Proliferative Indices,Apoptotic Indices, Prognostic Indicator

\section{Introduction}

Lymphoid neoplasms include various group of tumors of T-cell, B-cell, and NK-cell origin. Non-Hodgkin lymphoma (NHL) is a collective term for a heterogeneous group of lymphoproliferative malignancies with differing patterns of behaviour and responses to treatment.

Worldwide lymphomas rank $6^{\text {th }}$ among all cancers. ${ }^{(1)}$. Non Hodgkin lymphoma is the fifth most common cancer in the world with an upsurge of incidence in India. In past 4 decades the incidence of lymphoma has been doubled and it continues to peak. NHL in comparison to Hodgkin disease has a far greater predilection to disseminate to extranodal sites and much less predictable. The prognosis depends on the stage, histologic type, and treatment.

In lymphomas, immunohistochemistry is used in classifying, sub-classifying and in predicting the prognosis of several classes of lymphomas.

For the non Hodgkin's lymphomas there are many different classifications. Recent WHO classification of lymphomas (2008) classifies NHL into B and T cell lymphomas and for this a panel of immunohistochemical markers are to be used but are cost effective.

So in a case series study done by Lorenzo leoncini et al (2) quoted that working formulation classification of lymphomas still holds a greater significance. But to date there is no single classification that can predict how a particular tumour in an individual patient might behave. So a need to look at other prognostic indicators becomes necessary. Hence in this study we took apoptotic and proliferative indices as prognostic indicators ${ }^{(3)}$ and we tried to evaluate the role of these indices in various subtypes of non-Hodgkin lymphomas classified by working formulations.

\section{Materials and Methods}

Study material includes cases of Non Hodgkin lymphoma diagnosed in the Department of Pathology of Tirunelveli Medical College during a period of 3 years from 2014 to 2017.The haematoxylin and eosin stained sections which were prepared from formalin fixed paraffin embedded blocks of all cases of non Hodgkin lymphoma are collected. 
Immunohistochemistry done for Ki67 on coated slides and proliferative indices are calculated.

Apoptotic bodies are better appreciated in thin sectioned slides. So a section of

3-4 microns thickness are made. Slides were stained with routine $\mathrm{H} \& \mathrm{E}$

stains. Apoptotic bodies are counted and graded. All cases are classified by

\section{working formulations into}

1. High grade of lymphoma

2. Intermediate grade of lymphoma

3. Low grade of lymphoma

All cases that were diagnosed as non-Hodgkin lymphoma, both nodal and extranodal non-Hodgkin lymphoma were included. Inadequate lymph node sample, autolysed specimen, cases with dense tissue necrosis were excluded.

\section{Result}

Thus, among the total 57 cases of NHL by working formulation classification 21 cases are of low grade,23 cases are intermediate grade and 13 cases are high grade (Table 1). And then by applying prognostic indicators like proliferation and apoptotic indices Out of the 21 cases of low grade lymphomas 17 cases show proliferation rate of less than $40 \%$ while the rest of the cases show proliferation rate of greater than $45 \%$ and these low grade lymphomas may behave as intermediate grade(Figure 1). And 18 cases show a low apoptotic indices of less than $2.4 \%$ while the rest of the cases show apoptotic indices greater than $2.4 \%$ (Figure 2).

Similarly, out of the 23 cases of intermediate grade lymphomas 13 cases show proliferation rate between $40-75 \%$ while among the rest of the 10 cases 5 cases show proliferation rate of less than $40 \%$ and these may behave as a low grade with good survival. The other 5 cases show proliferation rate of greater than $80 \%$ and these intermediate grade lymphomas may behave as a higher grade with poor survival. There is a great variation in apoptotic indices of intermediate grade lymphomas (Figure 3). Out of the 23 cases 12 cases show apoptotic indices of greater than $2.4 \%$ while rest of the 11 cases show apoptotic indices of less than $2.4 \%$ (Figure 4 )

Among the 13 cases of high grade lymphoma all the cases shows a very higher proliferative capacity (Figure 5) and 10 cases show high apoptotic indices of greater than $2.4 \%$ while rest of the 3 cases show lower apoptotic indices of less than $2.4 \%$ (Figure 6 ). These all may behave as a really very aggressive tumour with a poorer survival rate. All the NHL cases with varying apoptotic and proliferative indices are calculated (Table 1) and the mean values of these indices are also tabulated (Table 2).

Table 1 : Average Values of Proliferative and Apoptotic Indices.

\begin{tabular}{|c|c|c|c|c|}
\hline $\begin{array}{c}\text { WORKING } \\
\text { FORMULATION }\end{array}$ & $\begin{array}{c}\text { PROLIFERATIVE } \\
\text { INDICES (MEAN } \\
\text { VALUE) }\end{array}$ & $\begin{array}{c}\text { STANDARD } \\
\text { DEVIATION }\end{array}$ & $\begin{array}{c}\text { APOPTOTIC INDICES } \\
\text { (MEAN VALUE) }\end{array}$ & $\begin{array}{c}\text { STANDARD } \\
\text { DEVIATION }\end{array}$ \\
\hline Low grade & 23.43 & 19.87 & 2.91 & 2.68 \\
\hline Intermediate & 56.91 & 23.00 & 2.22 & 2.41 \\
\hline High grade & 88.15 & 4.14 & 0.56 & 0.15 \\
\hline
\end{tabular}

Table 2 :Observation.

\begin{tabular}{|c|c|c|c|c|c|}
\hline \multicolumn{2}{|c|}{ Low } & \multicolumn{4}{c|}{ OBSERVATION } \\
\cline { 2 - 6 } & Intermediate & High & \multicolumn{2}{c|}{ Total } \\
\hline $\begin{array}{c}\text { WORKING } \\
\text { FORMULATION } \\
\text { CLASSIFICATION }\end{array}$ & Low grade & 17 & 4 & 0 & 21 \\
\cline { 2 - 6 } & Intermediate grade & 4 & 14 & 13 & 23 \\
\cline { 2 - 6 } & High grade & 0 & 13 & 18 & 57 \\
\hline
\end{tabular}




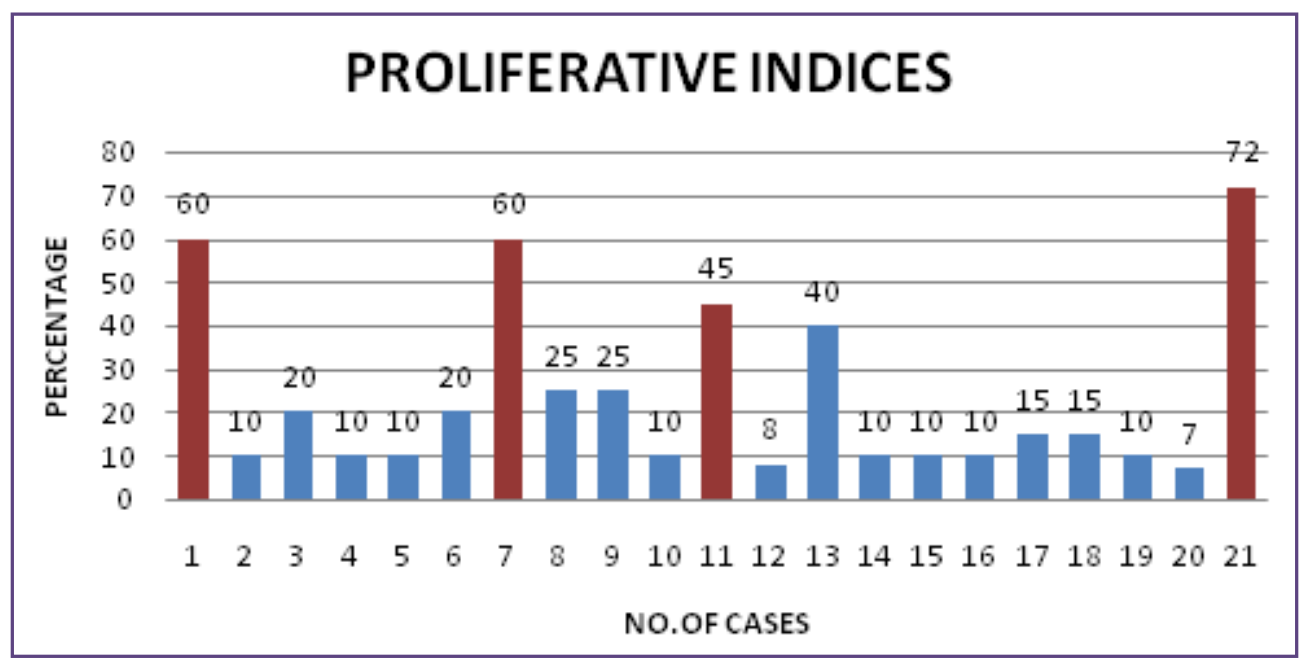

Fig. 1: Low Grade Lymphoma.

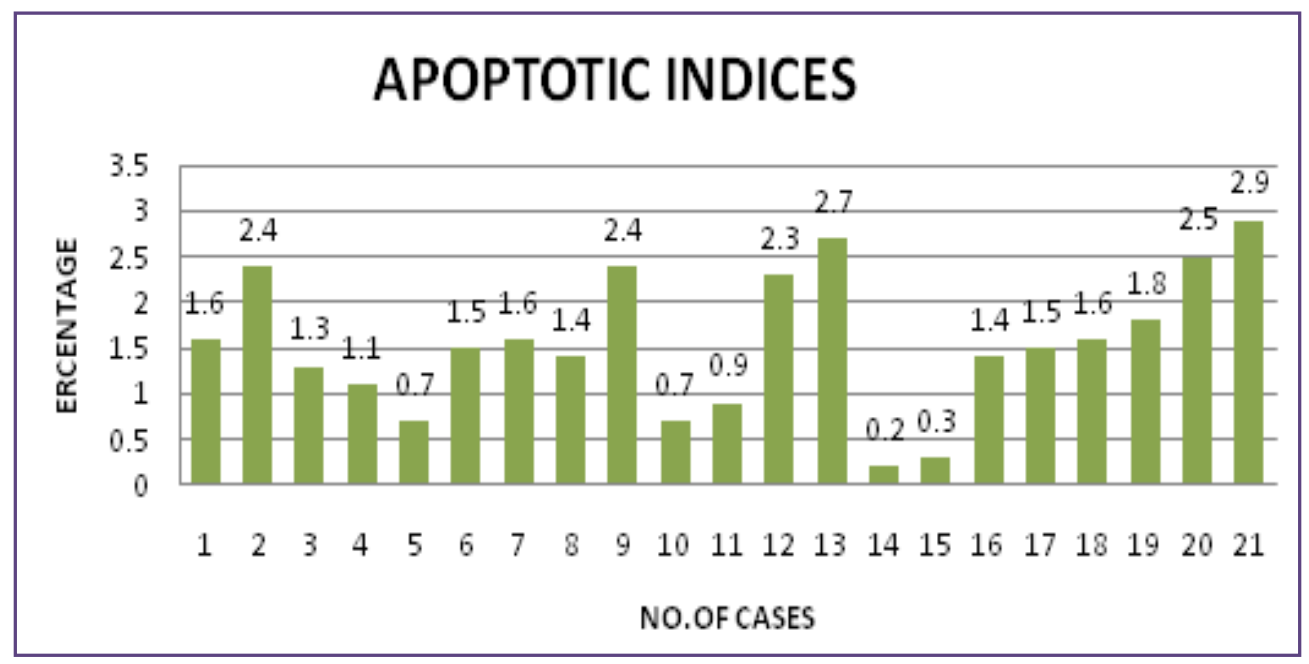

Fig. 2: Low Grade Lymphoma.

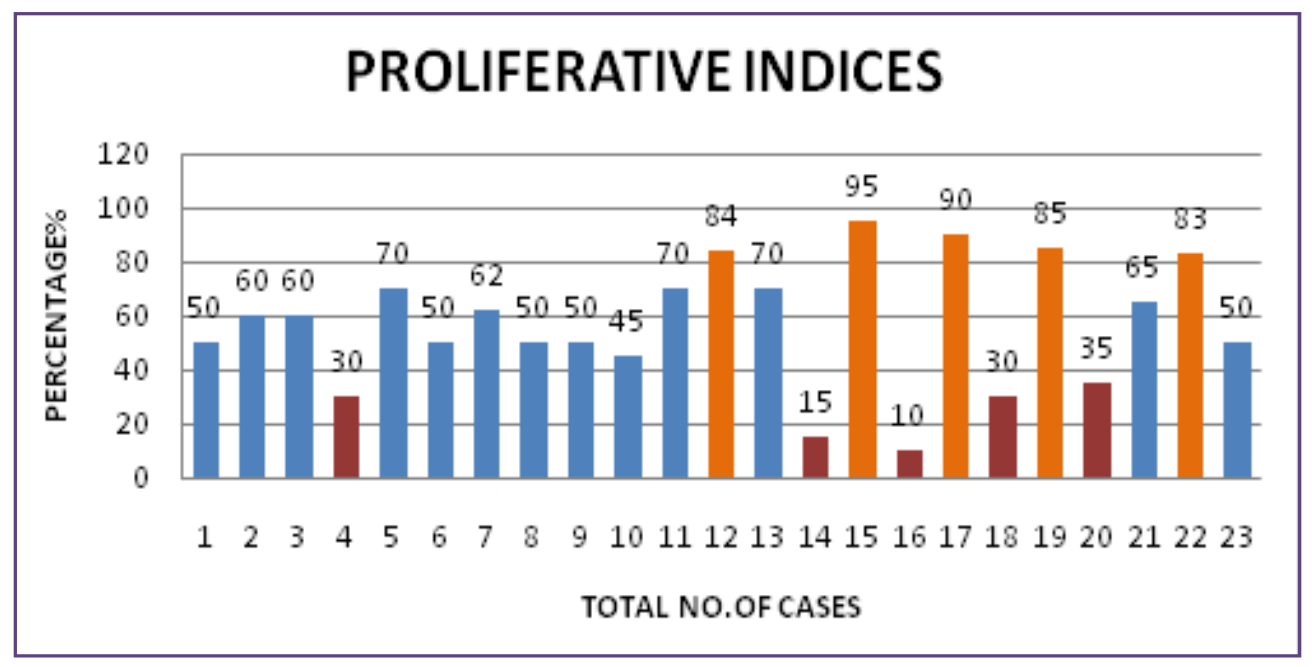

Fig. 3: Intermediate Grade Of Lymphomas. 


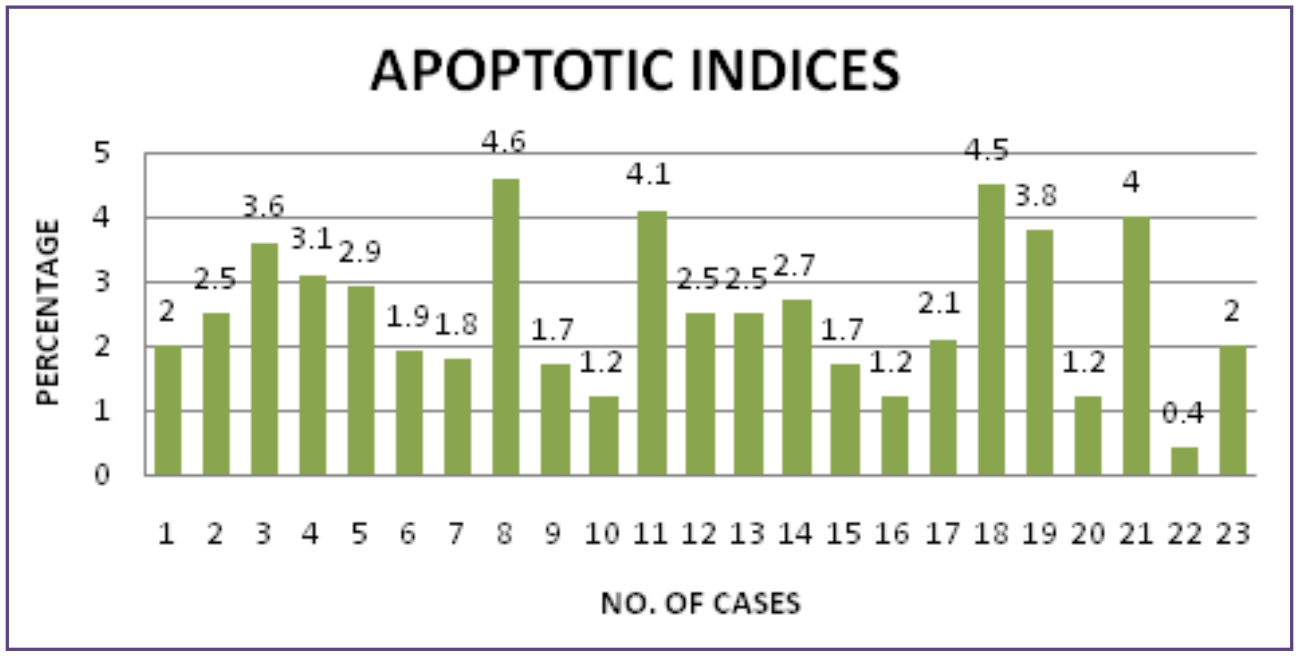

Fig. 4 : Intermediate Grade of Lymphoma.

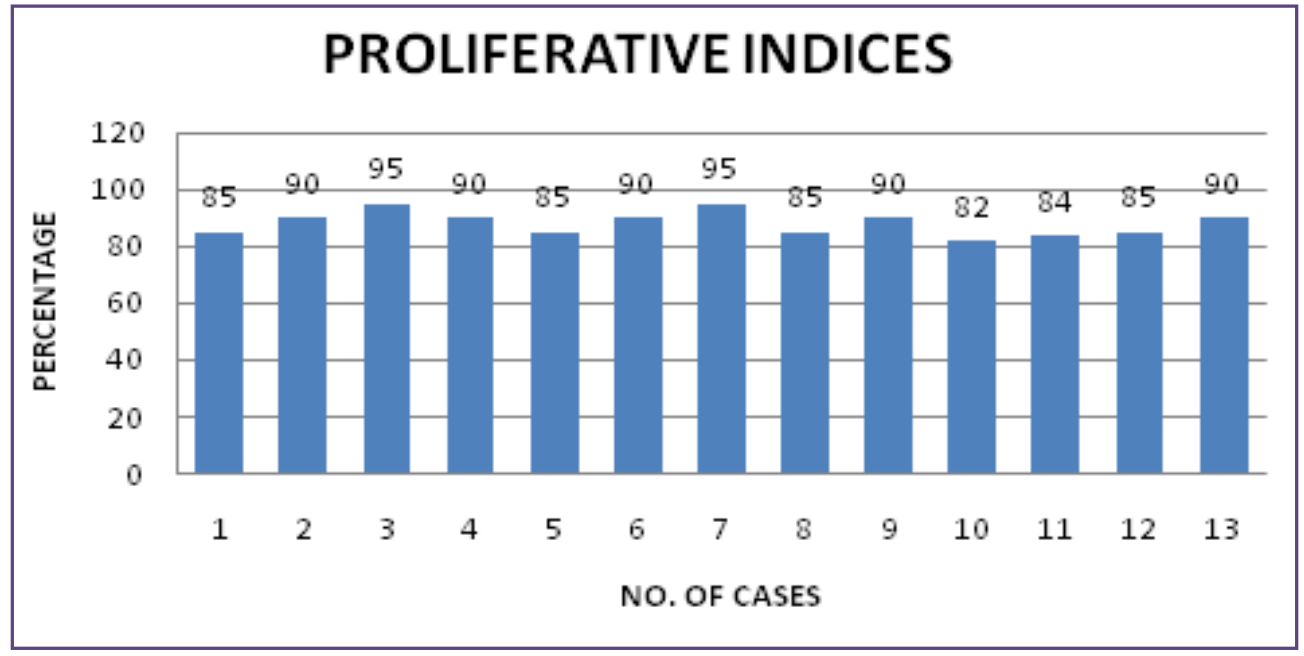

Fig.5 : High Grade Lymphomas.

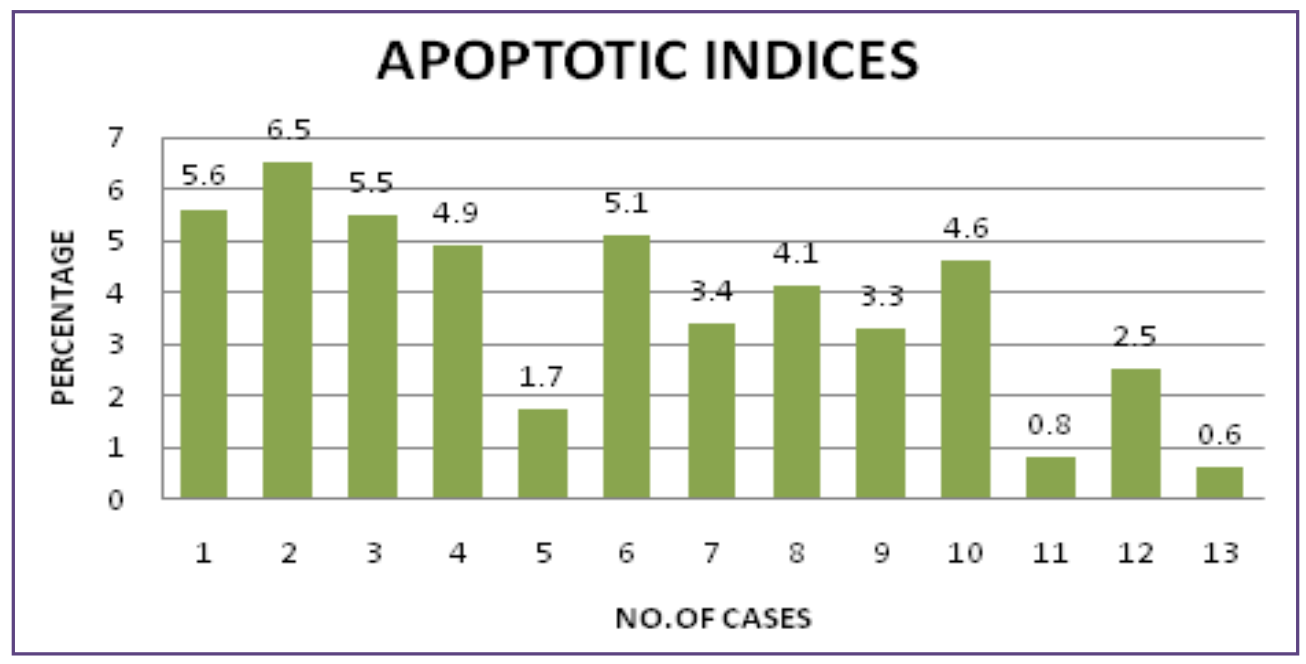

Fig. 6: High Grade Lymphomas. 


\section{Discussion}

Cell proliferation rate and death rate is an important factor for grading

human neoplasms and predicting their clinical behaviour and outcome. This study aimed at the examination of the prognostic significance of $\mathrm{Ki}-67$ expression and apoptotic indices in malignant non-Hodgkin's lymphomas.

Though numerous classifications for non-Hodgkin lymphomas were available no single classification determines how a tumour behave in a particular patient. Though WHO classification holds good, a minimum of 6 immunohistochemical markers are required for the classification which is expensive. So in this study we classified NHL by working formulations and assessed the trustworthiness of this classification by applying prognostic indicators like proliferative and apoptotic indices.

We demonstrated the Ki-67 expression in tumour cells by calculation of the proportion of immunopositive cells. This allows the determination of the proliferative activity of the tumour.

Adi broyde et $\mathrm{al}^{(4)}$ in his study mentioned that low /indolent grade lymphomas show a Ki67 proliferation rate at an average of about $27 \%$. In our study out of 21 low grade lymphomas 17 cases show $\mathrm{Ki}-67$ proliferation rate at less than $40 \%$. Only 4 cases of low grade lymphoma show proliferation rate between $45-75 \%$.

Zeggai et $\mathrm{al}^{(5)}$ in his study mentioned that aggressive / intermediate grade lymphomas show a Ki67 proliferation rate ranges between $40-75 \%$. In our study 23 cases were of intermediate grade lymphomas. Among that 13 cases show proliferation rate between $45-75 \%$ while 5 cases show a proliferation rate greater than $80 \%$. Remaining 5 cases show a proliferation rate of less than $35 \%$.

Olga boycov et al ${ }^{(6)}$ in his study mentioned that very aggressive /high grade lymphomas show a proliferation rate of $>80 \%$. In our study all high grade lymphomas show proliferation rate of $>80 \%$.

Leoncini et al ${ }^{(7)}$ in his study mentioned that low grade lymphomas show a lower apoptotic indices $<2.4 \%$ In our study out of 21 low grade lymphomas 18 cases show apoptotic indices of less than $2.4 \%$ and 3 cases show apoptotic indices greater than $2.4 \%$

Soini et $\mathrm{al}^{(8)}$ in his study on "histopathological evaluvation of apoptosis in cancer" mentioned that there is variation in apoptotic indices in varying grades of lymphomas. He also stated that high grade lymphomas and intermediate grade lymphomas show wide range of apoptotic indices from 2.5-8.8. Leoncini et al in his study also mentioned that there was a tendency for greater dispersion towards higher Apoptotic indices values in high grade than in intermediate grade NHLs. In our study out of 23 cases of intermediate grade lymphomas 12 cases show apoptotic indices value greater than $2.4 \%$ and the remaining 11 cases show a value less than $2.4 \%$.Roser et al. ${ }^{(9)}$ in his study mentioned that apoptotic index has shown an direct correlation with the proliferative index, corresponding to a more aggressive clinical course of the disease. In our study out of 13 cases of high grade lymphomas only 3 cases show apoptotic indices value of less than $2.4 \%$ and the remaining 10 cases show apoptotic indices value ranging from $2.5-6.5 \%$

Gerdes et al in his study have indicated a relationship between expression levels of $\mathrm{Ki}-67$ on one hand and the histological type of the tumour, which affects the degree of malignancy, and survival time in NHL on the other ${ }^{(10,11,12)}$. Tiemann et al. (13), who have shown that in patients with diagnosed high grade lymphoma with high proliferation index has correlated with shorter survival time. Martin et al. ${ }^{(14)}$, examining low grade lymphomas, also found that a lower proliferative index was associated with longer survival time was observed.

\section{Conclusion}

Thus by concluding, working formulation classification of Non Hodgkin lymphoma holds good for many cases it has its drawbacks in some cases. So it is ineffective to use it as a prime classification for Non Hodgkin lymphoma. Though apoptotic indices were found not to be significant in this study,proliferative indices were found to play an important in identifying the aggressiveness of tumour behaviour. Thus by the use of inexpensive and an effective prognostic indicators along with the working formulation classification it may throw some light on how a particular subtypes of Non hodgkin lymphoma in a particular patient might behave.. The outcome of the analysis of these indices has a positive correlation and has a definite impact over the prognosis of patients with NHL.

\section{References}

1. Rao SI. Role of immunohistochemistry in lymphoma, Indian J Med PaediatrOncol. 2010;31(4):145-147.

2. Del Vecchio MT, Leoncini L, Buerki K, Kraft R, Megha T, Barbini P, Tosi P, Cottier H: Diffuse centrocytic and/ or centroblastic malignant non-Hodgkin's lymphomas: comparison of mitotic and pyknotic (apoptotic) indices. Int J Cancer 1991, 47:38-43

3. Hall PA, Woods AL: Immunohistochemical markers of cellular proliferation: achievements, problems and prospects. Cell Tissue Kinet 1990, 23:505-522

4. Olga B, Yulia S, Elimelech O, Ofer S, and Osnat B. Role and prognostic significance of the Ki-67 index in non-Hodgkin's lymphoma. Am. J. H, 2009 .84: 338-343. 
5. Soumia Z, Noria H, Abdelnacer T, Feriel S, Meriem N. $\mathrm{M}$, Rachida S. Immunohistochemistry and scoring of Ki67 proliferative index and p53 expression in gastric B cell lymphoma from Northern African population: a pilot study: Journal of Gastrointestinal Oncology $2016: 462-469$

6. Olga B, Yulia S, Elimelech O, Ofer S, and Osnat B. Role and prognostic significance of the Ki-67 index in non-Hodgkin's Lymphoma: Am. J. Hematol .84: 338-343,2009.

7. Lorenzo L, Maria T, Del V, Tiziana M, Paolo B, Piero G, Stefano P, Elena S, Filippo G, Piero T, Rainer K, and Hans C: Correlations Between Apoptotic and Proliferative Indices in Malignant Non-Hodgkin's Lymphomas- American Journal of Pathology, 1993 755-762.

8. Y. Soini, P. Pa"a"kko“ , V-P. Lehto: Histopathological Evaluation of Apoptosis in Cancer: American Journal of Pathology, 1998;1041-1053

9. Roser F, Saini M, Meliss R, Ostertag H, Samii M and Bellinzona M: Apoptosis, vascularity, and proliferation in primary central nervous system lymphomas (PCNSL): a histopathological study. Surg Neurol 2004;62(5): 393-399.

10. Gerdes $\mathrm{J}$ : Ki-67 and other proliferation markers useful for immunohistologocal diagnostic and prognostic evaluations in human malignancies. Semin Cancer Biol 1:1990; 199-206.
11. Hall PA, Richards MA, Gregory WM, d'Ardenne AJ, Lister TA and Stansfeld AG: The prognostic value of Ki67 immunostaining in non-Hodgkin's lymphoma. J Pathol 1998;154: 223-235.

12. Velders GA, Kluin-Nelemans JC, De Boer CJ, Hermans J, Noodijk EM, Schuuring E, Kramer Mh, Van Deijk WA, Rahder JB, Kluin PM and Van Krieken JH: Mantle-cell lymphoma: a populationbased clinical study. J Clin Oncol 14(4): 1269-1274, 1996.

13. Tiemann M, Schrader C, Klapper W, Dreyling MH, Campo E, Norton A, Berger F, Kluin P, Ott G, Pileri S, Pedrinis E, Feller AC, Merz H, Janssen D, Hansmann ML, Krieken H, Moller P, Stein H, Unterhalt M, Hiddemann W and Parwaresch R: Histopathology, cell proliferation indices and clinical outcome in 304 patients with mantle cell lymphoma (MCL): a clinicopathological study from the European MCL Network. Br J Haematol 2005;131: 29-38.

14. Martin AR, Weisenburger DD, Chan WC, Ruby EI, Anderson JR, Vose JM, Bierman PJ, Bast MA, Daley DT and Armitage JO: Prognostic value of cellular proliferation and histologic grade in follicular lymphoma. Blood 1995;85: 3671- 3678 .

*Corresponding author:

Dr. K. Shantaraman, Professor and HOD, Department of Pathology, Tirunelveli Medical College, Tirunelveli- 627011 Tamilnadu, India

Phone: +91 9443133898

Email: shantaraman_kal@tvmc.ac.in

Financial or other Competing Interests: None. 